\title{
A SPATIAL STUDY ON KESHAN DISEASE PREVALENCE AND SELENOPROTEIN P IN THE HEILONGJIANG PROVINCE, CHINA
}

\author{
YANAN WANG ${ }^{1}$, XIAO ZHANG ${ }^{1,2}$, TONG WANG ${ }^{1}$, JIE HOU ${ }^{1}$, ZHONGYING GUO ${ }^{1,3}$, XIAOMIN HAN $^{1,3}$, \\ HUIHUI ZHOU ${ }^{1,4}$, HONG LIANG $^{1,5}$, and ZHIFENG XING ${ }^{6}$
}

${ }^{1}$ Harbin Medical University, Harbin, China

Institute of Keshan Disease, Chinese Center for Endemic Disease Control

${ }^{2}$ Qingdao Municipal Hospital, Qingdao, China

Department of Healthcare-Associated Infection Management

${ }^{3}$ Harbin Center for Disease Control and Prevention, Harbin, China

Director Office

${ }^{4}$ Jining Medical University, Jining, China

School of Public Health

${ }^{5}$ Qiqihar Medical University, Qiqihar, China

School of Public Health

${ }^{6}$ Heilongjiang Center for Disease Control and Prevention, Harbin, China

Institute for Endemic Disease Control and Prevention

\begin{abstract}
Objectives: Few spatial studies on Keshan disease (KD) prevalence and serum selenoprotein P (SELENOP) levels have been reported in the Heilongjiang Province, China. This study aimed to investigate the spatial relationships between KD prevalence, SELENOP levels, and the socio-economic status for the precise prevention and control of KD. Material and Methods: The study was carried out in all the $66 \mathrm{KD}$ endemic counties in the Heilongiiang Province using a non-probability sampling method of a key village survey based on county-wide case-searching. The participants completed a questionnaire and had their serum SELENOP levels measured using enzyme-linked immunosorbent assay. Thematic maps were created, and spatial regression analysis was performed by ordinary least squares using ArcGIS 9.0. Results: Overall, 53676 residents were surveyed based on case-searching, and blood samples were collected from 409 residents. In total, 50 chronic KD cases were identified with a total prevalence of $9.3 / 10000$ population. The prevalence in the Tangyuan County was the highest (250/10 000 population). The mean serum SELENOP level was $13.96 \mathrm{mg} / \mathrm{l}$. The spatial regression analysis showed that KD prevalence positively correlated with SELENOP levels and negatively with per capita disposable income among rural residents. Conclusions: The Tangyuan County should be considered for the precise prevention and control of KD. Further research is necessary to verify the reliability of SELENOP for estimating body selenium levels, and to better understand the relationship between selenium intake and KD in the investigated area. Int J Occup Med Environ Health. 2021;34(5):659-66
\end{abstract}

Key words:

prevalence, biomarker, prevention and control, endemic cardiomyopathy, selenoprotein $\mathbf{P}$, spatial regression analysis

Funding: this work was supported by the National Natural Science Foundation of China (grant No. 82073492 entitled "Spatial Epidemiological Study of Keshan Disease at Township Level in China," grant manager: Prof. Tong Wang) and by the National Natural Science Foundation of China (grant No. 81773368 entitled "A Spatial Epidemiological Study of Keshan Disease," grant manager: Prof. Tong Wang).

Received: October 12, 2020. Accepted: February 8, 2021.

Corresponding author: Tong Wang, Harbin Medical University, Institute of Keshan Disease, Chinese Center for Endemic Disease Control, 157 Baojian Road, Harbin 150081, P.R. China (e-mail: wangtong@ems.hrbmu.edu.cn). 


\section{INTRODUCTION}

Keshan disease (KD), an endemic cardiomyopathy characterized primarily by myocardial injuries, was first reported in the Keshan County, the Heilongjiang Province, China [1-3]. The Heilongjiang Province where KD was the most prevalent in the 1950s through 1960s has $66 \mathrm{KD}$ endemic counties, accounting for $20 \%$ of the total KD endemic counties in China [4,5]. Since late 1970s, both the incidence and prevalence of KD have declined, and the disease has been well controlled [6,7].

Many observational and experimental studies have shown that selenium deficiency is a cause of $\mathrm{KD}$ based on causation of modern epidemiology [8-11]. Selenium plays a biological role in the form of selenoproteins in the human body, of which the selenium content bound by selenoprotein P (SELENOP) is the highest, reflecting the best selenium nutrition of the human body [12,13]. Not only is serum SELENOP considered the best measure of selenium containing proteins, but it can also be used to evaluate the effectiveness of KD prevention and control measures. Because of the endemicity of $\mathrm{KD}$, spatial epidemiology may provide some useful prevention and control strategies by elucidating spatial characteristics and identifying the clustering of $\mathrm{KD}$ [14]. Although the authors' team had previously carried out a spatial description and analysis of the serum SELENOP levels in residents living in rural and urban areas in the KD endemic and non-endemic provinces in mainland China, KD prevalence and serum SELENOP levels were not closely examined at the county level [15]. Therefore, this study aimed to understand and visualize the current prevalence of KD and its influencing factors to provide evidence for the precise prevention and control of $\mathrm{KD}$ at the county level in the Heilongjiang Province.

\section{MATERIAL AND METHODS}

\section{Study design}

The study was carried out in all $66 \mathrm{KD}$ endemic counties in the Heilongjiang Province using a non-probability sam- pling method of a key village survey based on county-wide case-searching according to the Implementation Project for Prevention and Control of Endemic Disease [16]. The method was similar to that described by Li [17]. First, case-searching was conducted by reviewing medical records in all county-, township-, and village-level medical institutions within the 66 counties. Second, the 2 townships with the greatest number of $\mathrm{KD}$ cases were selected from each county. Third, 1 village with the greatest number of $\mathrm{KD}$ cases was selected from each township as a key survey site. All permanent residents within the survey site underwent medical examinations, and completed questionnaires. The reported KD cases confirmed or suspected by village doctors were identified using a combination of the medical history, clinical characteristics, and epidemiological data based on the Criteria for Diagnosis of Keshan Disease [1] at the township or county hospitals. The study was approved by the Ethical Committee Review Board of Harbin Medical University (hrbmuecdc20200302, hrbmuecdc20180301).

\section{Survey areas}

All the 66 endemic counties, including Acheng, Aihui, Anda, Bayan, Baiquan, Baoqing, Beian, Bin, Boli, Didao, Dongning, Dongshan, Duerbote, Fangzheng, Fujin, Fuyu, Gannan, Hailin, Hailun, Huma, Hulin, Huachuan, Huanan, Jidong, Jixian, Jiayin, Jiaoqu, Kedong, Keshan, Lanxi, Lindian, Linkou, Lingdong, Longjiang, Mashan, Meilisi, Mishan, Mingshui, Mulan, Muling, Nehe, Nenjiang, Ning'an, Qiezihe, Qinggang, Qing'an, Raohe, Saertu, Shangzhi, Sifangtai, Suibin, Suifenhe, Suiling, Sunwu, Tahe, Tangyuan, Taoshan, Tieli, Tonghe, Wuchang, Wudalianchi, Xunke, Yanshou, Yi'an, Yilan, and Zhaodong, were surveyed in the Heilongjiang Province.

\section{Population surveyed}

In the selected $\mathrm{KD}$ endemic areas, all permanent residents, defined as those who lived in their residence for $\geq 6$ months every year, were asked to participate. The inclusion crite- 
ria for the participants with serum SELENOP measured were as follows: age 17-30 years, ability to converse with researchers, volunteering to join the study, and willingness to provide written informed consent.

\section{Questionnaire}

The following basic epidemiological data were obtained from a pre-designed questionnaire, which included questions on gender, age, a history of $\mathrm{KD}$, and address.

\section{Criteria}

The suspected cases of KD were diagnosed according to the Criteria for Diagnosis of Keshan Disease (WS/T 2102011) [1]. The evaluation of disease prevention and control was based on factors included in the Delimitation and Classification of Keshan Disease Areas (GB 17020-2010) and the Evaluation of Key Endemic Disease Control and Elimination (2019) criteria [18,19].

\section{Serum SELENOP}

From each of the 409 subjects, a sample of $2 \mathrm{ml}$ of venous blood was collected after they had fasted for $8 \mathrm{~h}$. The serum was separated and stored in a refrigerator at $-80^{\circ} \mathrm{C}$ for analysis. The serum SELENOP was measured using enzyme-linked immunosorbent assay kits (Shanghai Enzyme-Linked Biotechnology Co., Ltd, Shanghai, China) according to the manufacturer's instructions.

\section{Per capita disposable income}

Data on per capita disposable income were collected through the Heilongjiang Statistical Yearbook [20].

\section{Statistical analyses}

Data input was conducted using Epi Info v. 3.5.1 (Centers for Disease Control, Atlanta, GA, USA). Statistical analyses were performed using SPSS v. 17.0 (IBM Corp, Armonk, NY, USA). The spatial descriptions and regression analyses of KD, SELENOP, and per capita disposable income were conducted by creating thematic maps and ordinary least squares using ArcGIS v. 9.0 (Esri, Redlands, CA, USA).

\section{RESULTS}

\section{Prevalence rate of KD}

In the study, 53676 study participants were investigated from among the permanent residents of the selected endemic areas. In total, 50 chronic $\mathrm{KD}$ cases were detected with a prevalence rate of 9.3/10 000 population (50/53 676). The cases of chronic KD were distributed among 8 counties including Shangzhi, Fuyu, Mishan, Lindian, Huanan, Tieli, Tangyuan, and Wudalianchi. Among the counties, the prevalence rate in the Tangyuan County (250/10 000 population) was the highest while the lowest prevalence rate was recorded in the Shangzhi County (12.2/10 000 population). The spatial distribution of the KD prevalence rate is shown in Figure 1.

\section{Mean serum SELENOP levels}

A total of 409 participants, including 120 (29.3\%) men and $289(70.7 \%)$ women, were included in this survey. The average age of the subjects was 22 years, including 151 (36.9\%) people aged $<20$ years, 192 (46.9\%) aged 20-24 years, and $66(16.1 \%)$ aged $>24$ years. The mean serum SELENOP level was similar in men $(13.9 \mathrm{mg} / \mathrm{l}, 95 \%$ confidence interval [CI]: $12.7-15.1)$ and women $(14.0 \mathrm{mg} / \mathrm{l}, 95 \% \mathrm{CI}$ : 13.1-14.8). The mean serum SELENOP level of the people living in the endemic areas was $13.96 \mathrm{mg} / \mathrm{l}$. The mean serum SELENOP level of the residents of the Meilisi County was the lowest $(5.6 \mathrm{mg} / \mathrm{l})$ while that of the residents of the Qiezihe County was the highest $(27.3 \mathrm{mg} / \mathrm{l})$. In $34 \mathrm{KD}$ endemic counties, including Acheng, Aihui, Baiquan, Binxian, Boli, Didao, Dongshan, Fangzheng, Gannan, Hailin, Hailun, Hulin, Huachuan, Jixian, Jiayin, Keshan, Lindian, Linkou, Meilisi, Muling, Nehe Nenjiang, Ning'an, Qing'an, Raohe, Shangzhi, Suibin, Suifenhe, Taoshan, Tonghe, Wuchang, Xunke, Yanshou, and Yian, the mean serum SELENOP 


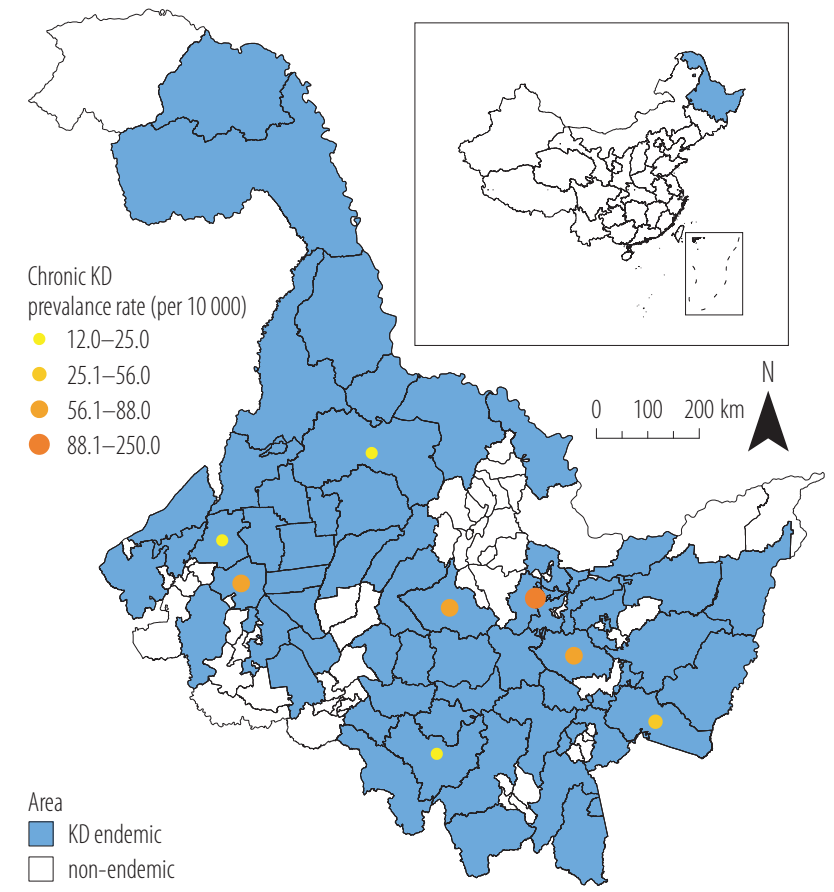

Figure 1. Spatial distribution of the Keshan disease (KD) prevalence rate in $\mathrm{KD}$ endemic counties in the Heilongiiang Province, China, in 2015-2016

levels were lower than the average value of $13.96 \mathrm{mg} / \mathrm{l}$, accounting for $51.5 \%(34 / 66)$ of all the endemic counties surveyed. The spatial distribution of the mean serum SELE$\mathrm{NOP}$ levels in $\mathrm{KD}$ endemic areas is shown in Figure 2.

\section{Per capita disposable income}

The spatial distribution of per capita disposable income among rural residents of the $\mathrm{KD}$ endemic areas is shown in Figure 3. The average per capita disposable income of rural residents in the Heilongjiang Province was CNY 10453. The per capita disposable income of rural residents in the Tangyuan County was the lowest (3427 yuan) and that of rural residents in the Dongning County was the highest (18 090 yuan).

\section{Spatial regression analysis}

A spatial regression model was established with KD prevalence as the dependent variable, and the SELENOP level

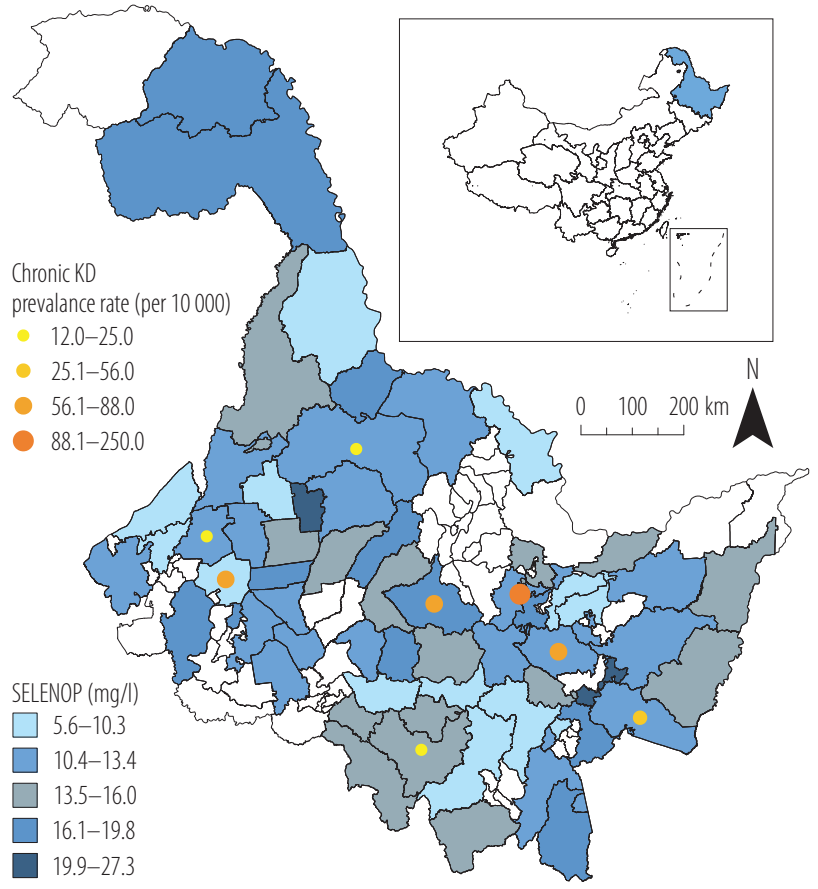

Figure 2. Spatial distribution of the mean serum selenoprotein $P$ (SELENOP) levels in Keshan disease (KD) endemic counties in the Heilongiiang Province, China, in 2015-2016

and per capita disposable income among rural residents as independent variables. The results are shown in Table 1. The independent variables were entered into the spatial regression model. The model residuals were tested using spatial autocorrelation analysis revealing that they were independent and had no spatial autocorrelation. The established ordinary least squares model was significant. The prevalence of KD positively correlated with the level of SELENOP and negatively with per capita disposable income among rural residents.

\section{DISCUSSION}

As $\mathrm{KD}$ is not a notifiable disease, its incidence or prevalence in the affected population can only be estimated through active surveillance. This case-searching method used in endemic counties is equivalent to a survey of the whole population when all the endemic counties are covered. At the same time, the authors found villages most 
affected by KD by combining methodologies for disease investigation in key populations. They focused on investigating the cofactors of the disease, as well as on monitoring and evaluating the prevention and control of $\mathrm{KD}$ in a holistic way [21]. This combined method has the advantages of timeliness, simplicity, flexibility, and improved data quality [22,23].

The survey sites covered $100 \%$ (66/66) of the KD endemic counties in the Heilongjiang Province. Therefore, the results are most likely to reflect the highest prevalence of $\mathrm{KD}$ and to indicate where further preventative measures need to be taken. As seen in Figure 1, 50 chronic KD cases were identified in only 8 counties, indicating that $\mathrm{KD}$ is well controlled. The highest KD prevalence was found in the Tangyuan County; thus, this should be the targeted area for KD prevention and control. The prevalence of KD in the Shangzhi County, historically considered a highly-affected area, was relatively low. Only the Shangzhi County, of the 8 counties with chronic cases, achieved the control goal but did not achieve the elimination goal according to the Content and Criteria of Keshan Disease Control and Elimination Assessment in the 2019 Key Endemic Disease Control and Elimination Assessment Method.

Compared with the prevalence of $\mathrm{KD}$ in Jilin, Liaoning, Shandong, Gansu, Inner Mongolia, Shaanxi, Shanxi, Hebei, and Henan, the prevalence of KD in the Heilongiang Province was lower [24]. These results suggest that,

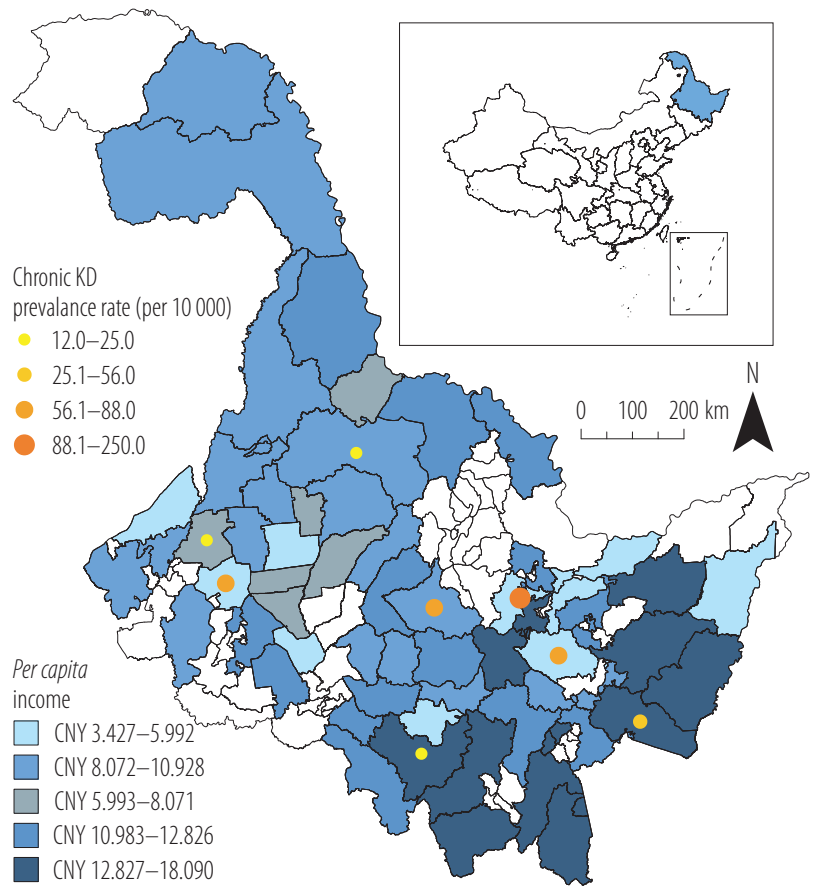

Figure 3. Spatial distribution of per capita disposable income among rural residents of Keshan disease (KD) endemic counties in the Heilongjiang Province, China, in 2015-2016

though the prevention and control measures for KD have achieved some success, the etiologic factors in some endemic areas may be still active. Therefore, it is necessary to continue the prevention and control of $\mathrm{KD}$.

The disease in question is an endemic cardiomyopathy, and its etiology is not fully understood. Since 1935, various etiological hypotheses have been proposed including the mycotoxin hypothesis, the selenium deficiency theory,

Table 1. Spatial regression analysis of the Keshan disease $(\mathrm{KD})$ prevalence rate in $\mathrm{KD}$ endemic counties in the Heilongjiang Province, China, in 2015-2016

\begin{tabular}{lccccc}
\hline \multicolumn{1}{c}{ Variable } & $\begin{array}{l}\text { Regression } \\
\text { coefficient }\end{array}$ & SD & $\mathrm{t}$ & $\mathrm{p}$ & $\mathrm{VIF}$ \\
\hline$\beta_{0}$ & 0.177 & 1.34 & 0.13 & 0.90 & - \\
SELENOP & 11.210 & 0.86 & 13.08 & $<0.001$ & 5.08 \\
Per capita disposable income & -0.010 & 0.001 & -7.58 & $<0.001$ & 5.08 \\
\hline
\end{tabular}

SELENOP - selenoprotein P; VIF - variance inflation factor.

$\mathrm{R}^{2}=0.67, \mathrm{R}_{\mathrm{adj}}{ }^{2}=0.66, \mathrm{~F}=129.12, \mathrm{p}<0.01$. 
and the virus theory. Among these, selenium deficiency is the best-supported theory. Assessing the association between $\mathrm{KD}$ and selenium deficiency is based on the principles of a causal inference of modern epidemiology.

As seen in Figure 2, the mean serum SELENOP levels among residents of 34 endemic counties, accounting for $51.5 \%(34 / 66)$ of the surveyed endemic counties, were lower than those in all endemic counties; these should be the key counties for KD prevention and control. As presented in Table 1, the spatial regression analysis showed that the prevalence of $\mathrm{KD}$ positively correlated with the level of serum SELENOP. Although the strong association of $\mathrm{KD}$ with selenium deficiency has been widely recognized, epidemiological studies on selenium risk doses in the development of KD remain poorly understood. Previous reports on the relationship between selenium and other indicators of human nutritional status and $\mathrm{KD}$ were inconsistent, especially the results of the cross-sectional studies. This study is also consistent with cardiovascular disease studies that have reported adverse effects [25-27].

The spatial regression analysis showed that the prevalence of KD negatively correlated with the level of per capita disposable income. The KD endemic areas are all rural areas, which are remote and mountainous, with inconvenient transportation and underdeveloped economy [28-30]. The diet of residents living in $\mathrm{KD}$ endemic areas was mainly affected by locally grown grains [3,31]. With economic development, a large proportion of grains with higher nutrient value has been imported; thus, with the resultant improvement in diet, selenium deficiency has remarkably decreased, and the prevalence of KD has declined. Therefore, rapid economic developments and substantial improvements in the living standards of residents have led to a decline in $\mathrm{KD}$ prevalence. The level of per capita disposable income in the Tangyuan County is the lowest in the province; thus, the Tangyuan County should be the focus of holis- tic interventions aimed at the prevention and control of KD through economic development.

Serum SELENOP is probably the biomarker that best represents selenium nutrition levels in the human body. It is undoubtedly of great significance to scientifically provide precise visual evidence to supplement molecular-level information on the KD etiology of selenium deficiency, and to provide evidence of the effectiveness of prevention and control measures. The results are valuable for KD prevention and control in terms of selenoprotein nutrition. This research translates the advances of selenoproteins and spatial epidemiology into KD prevention and control. In addition, its results can be used as evidence for the selenium nutritional level of residents in the different counties of the Heilongiiang Province. Some limitations of this study include the small numbers of participants who had their serum SELENOP assessed, the low proportion of residents living in some of the KD endemic areas, and the lack of selenium measurements to verify SELENOP data. An additional selenium biomarker measure would be helpful to improve the quality of collected data. Further molecular-level investigations to understand the value of different selenium biomarkers are warranted [32,33]. The authors wish to further explore the relationship between plasma or serum selenium, hair selenium, and SELENOP levels.

\section{CONCLUSIONS}

The Tangyuan County should be considered for the precise prevention and control of KD. Further research is necessary to verify the reliability of SELENOP for estimating body selenium levels, and to better understand the relationship between selenium intake and $\mathrm{KD}$ in the investigated area.

\section{ACKNOWLEDGMENTS}

The authors would like to thank the study participants for their contribution to the research, as well as investigators and staff. 


\section{REFERENCES}

1. National Health Commission of the People's Republic of China [Internet]. Beijing: Standards Press of China; 2011 [cited 2011 Apr 26]. The Criteria for Diagnosis of Keshan Disease (WS/T 210-2011). Available from: http://www.nhc. gov.cn/wjw/s9500/wsbz.shtml.

2. Xu GL. Research progress of prevention of Keshan disease by supplementation of selenium and the relationship between selenium deficiency and Keshan disease: commemorating the 60th anniversary of the discovery of Keshan disease. Endemic Dis Bull. 1996;11(2):1-6.

3. Yang GQ, Wang GY, Yin TA, Sun SZ, Zhou RH, Man RE, et al. Relationship between Keshan Disease Distribution and Selenium Nutrition Condition in China. Acta Nutrimenta Sinica. 1982;4(3):191-200.

4. Li Q, Liu M, Hou J, Jiang C, Li S, Wang T. The prevalence of Keshan disease in China. Int J Cardio. 2013;168(2):1121-6, https://doi.org/10.1016/j.ijcard.2012.11.046.

5. Disease Prevention and Control Bureau of the National Health Commission. Questionnaire on Prevention and Control of Keshan Disease. Beijing: The Bureau; 2017.

6. Zhang ZG, Li XY, Fu D, Zhou SB, Pan HD, Tian YM, et al. Surveillance Report of Keshan Disease in Heilongiiang Province. Chin J Endemiol. 1989;8(5):308-11.

7. Wang T, Hou J, Li Q, Zhang LJ, Li XZ, Gao L, et al. Analysis of the data of national Keshan disease surveillance in 2004. Chin J Endemiol. 2005;24(4):401-3.

8. Xia YM, Hill KE, Burk RF. Biochemical studies of a selenium-deficient population in China: measurement of selenium, glutathione peroxidase and other oxidant defense indices in blood. J Nutr. 1989;119(9):1318-26, https://doi.org/10.1093/ jn/119.9.1318.

9. Gao XZ, Liu ZC, Wang T, Li Q, Liang N, Hou J, et al. Randomized trial of the effect of selenium supplementation on the progression of heart failure in chronic Keshan disease. Chin J Endemiol. 2016;3:241-4.

10. Hou J, Wang T, Liu MF, Li SE, Chen J, Liu C, et al. Suboptimal selenium supply-a continuing problem in Keshan disease areas in Heilongiang province. Biol Trace Elem Res. 2011;143(3): 1255-63, https://doi.org/10.1007/s12011-011-8961-9.

11. Zhou HH, Wang T, Li Q, Li DD. Prevention of Keshan Disease by Selenium Supplementation-systemic review and Meta-analysis. Biol Trace Elem Res. 2018;186(1):98-105, https://doi.org/10.1007/s12011-018-1302-5.

12. Steinbrenner H, Speckmann B, Klotz LO. Selenoproteins: Antioxidant selenoenzymes and beyond. Arch Biochem Bioph. 2016;595:113-9, https://doi.org/10.1016/j.abb.2015.06. 024.

13. Barrett CW, Short SP, Williams CS. Selenoproteins and oxidative stress-induced inflammatory tumorigenesis in the gut. Cell Mol Life Sci. 2017;74(4):607-16, https://doi.org/10.1007/ s00018-016-2339-2.

14. Beale L, Abellan JJ, Hodgson S, Jarup L. Methodologic issues and approaches to spatial epidemiology. Environ Health Perspect. 2008;116(8):1105-10, https://doi.org/10.1289/ehp. 10816.

15. Zhang X, Wang T, Li SE, Ye C, Hou J, Li Q, et al. A spatial ecological study of selenoprotein $\mathrm{P}$ and Keshan disease. J Trace Elem Med Bio. 2019;51:150-8, https://doi.org/10. 1016/j.jtemb.2018.10.011

16. Center for Endemic Disease Control of Chinese Center for Disease Control and Prevention [Internet]. Harbin: 2012 [cited 2012 Jul 10]. Implementation Project for Prevention and Control of Endemic Disease in 2012. Available from: http://210.46.80.45/crcfedc/zzxm-2.html.

17. Li SE, Wang T, Ye C, Li Q, Guo ZD, Wu H, et al. An approach to assessment of Keshan disease elimination at the township level. Int Health. 2016;8(6):398-404, https://doi. org/10.1093/inthealth/ihw045.

18. National Health Commission of the People's Republic of China [Internet]. Beijing: Standards Press of China; 2011 [cited 2011 Jan 14]. Delimitation and Classification of Keshan Disease Areas (GB17020-2010). Available from: http://www.nhc.gov.cn/wjw/s9500/wsbz_2.shtml.

19. National Health Commission of the People's Republic of China [Internet]. Beijing: 2019 [cited 2019 Jul 23]. Evaluation 
of Key Endemic Disease Control and Elimination in 2019. Available from: http://www.nhc.gov.cn/jkj/s5873/201908/b0ac881a9c5348e0aa3763fa2bc98d43.shtml.

20. Heilongjiang Bureau of Statistics [Internet]. Harbin: 2016 [cited 2016 Jan 26]. Heilongjiang Statistical Yearbook in 2015. Available from: http://jj.hlj.gov.cn/tjsj/tjnj/.

21. Taboy CH, Chapman W, Albetkova A, Kennedy S, Rayfield MA. Integrated Disease Investigations and Surveillance planning: a systems approach to strengthening national surveillance and detection of events of public health importance in support of the International Health Regulations. BMC Public Health. 2010;10(Suppl 1):S6, https:/doi. org/10.1186/1471-2458-10-S1-S6.

22. German RR, Lee LM, Horan JM, Milstein RL, Pertowski CA, Waller MN. Updated guidelines for evaluating public health surveillance systems: recommendations from the Guidelines Working Group. MMWR Recomm Rep. 2001; 50(RR-13):1-35.

23. Khoury MJ, Gwinn M, Ioannidis JP. The emergence of translational epidemiology: from scientific discovery to population health impact. Am J Epidemiol. 2010;172(5):517-24, https://doi.org/10.1093/aje/kwq211.

24. Guo ZY, Wang T, Han XM, Hou J, Wang YN, Duan YN, et al. Descriptive spatial epidemiology of Keshan disease prevention and control in China. Chin J Endemiol. 2018; 37(3):235-8.

25. Gore F, Fawell J, Bartram J. Too much or too little? A review of the conundrum of selenium. J Water Health. 2010;8(3): 405-16, https://doi.org/10.2166/wh.2009.060.
26. Bleys J, Navas-Acien A, Laclaustra M, Pastor-Barriuso R, Menke A, Ordovas J, et al. Serum selenium and peripheral arterial disease: results from the national health and nutrition examination survey, 2003-2004. Am J Epidemiol. 2009;169(8):996-1003, https://doi.org/10.1093/aje/kwn414.

27. Bleys J, Navas-Acien A, Guallar E. Serum selenium levels and all-cause, cancer, and cardiovascular mortality among US adults. Arch Intern Med. 2008;168(4):404-10, https:// doi.org/10.1001/archinternmed.2007.74.

28. The National Surveillance Group for Keshan Disease. Analysis of the data of national Keshan disease surveillance in 2007. Chin J Endemiol. 2008;27(4):412-5.

29. Wang $\mathrm{T}$. The translational epidemiology of Keshan disease surveillance. Foreign Med Sci (Sect Medgeogr). 2012;33(3): 143-7.

30. Wang T. Assessment of Keshan disease elimination: the challenges and opportunities. Chin J Endemiol. 2015;34(6): 391-2.

31. Xu GL, Xue WL, Zhang PY, Feng CF, Hong SY, Liang WS. Dietary selenium and body selenium level of residents in Keshan disease endemic and non-endemic areas. Acta Nutrimenta Sinica. 1982;4(3):183-90.

32. Muzembo BA, Dumavibhat N, Ngatu NR, Eitoku M, Hirota $\mathrm{R}$, Kondo $\mathrm{S}$, et al. Serum selenium and selenoprotein $\mathrm{P}$ in patients with silicosis. J Trace Elem Med Biol. 2013;27(1): 40-4, https://doi.org/10.1016/j.jtemb.2012.05.003.

33. Tsutsumi R, Saito Y. Selenoprotein P; P for Plasma, Prognosis, Prophylaxis, and More. Biol Pharm Bull. 2020;43(3): 366-74, https://doi.org/10.1248/bpb.b19-00837.

This work is available in Open Access model and licensed under a Creative Commons Attribution-NonCommercial 3.0 Poland License - http://creativecommons.org/ licenses/by-nc/3.0/pl/deed.en. 